\title{
$\nabla$ \\ IJCRR \\ Section: Healthcare \\ Comparative and Mechanistic Study on the Anti- Cancer Potentiality of Lycopersicon Esculentum Fruits and Cassia Fistula Pods in Breast Cancer
}

ISI Impact Factor

(2019-20): 1.628

IC Value (2019): 90.81

$\operatorname{SJIF}(2020)=7.893$

(c) (i) (3)

Copyright@IJCRR

\author{
Ajmire Prashant ${ }^{1 *}$, Patel Rakesh ${ }^{1}$, Kayande Nandu' ${ }^{2}$, Bakal Ravindra ${ }^{3}$
}

'School of Pharmacy, Dr. A. P. J. Abdul Kalam University, Indore (M. P), India; 'Thakur Shivkumar Singh Memorial Pharmacy College, Burhanpur, (M. P), India; 'ंDr. Rajendra Gode Institute of Pharmacy, Amravati, Maharashtra, India.

\section{ABSTRACT}

Introduction: High-throughput profiling of cancer tissue specimens and body fluids has been used extensively to reveal specific molecular fingerprints of breast cancer. Women with breast cancer are associated with several modifiable and non-modifiable risk factors. Despite the major advances made in the field of breast cancer screening and therapeutics, it remains a major health issue and a key cause of mortality for women across the globe. Especially, the off-target properties of chemotherapeutic agents remain the main concern. Being a good potential chemotherapeutic, the compound should target the rapidly dividing cancer cells, eventually create less cytotoxicity to normal cells.

Aim: To do so, in our present study, we have studied the anti-cancer potentiality of Lycopersicon esculentum fruits and Cassia fistula pods in vitro breast cancer cells and compared their anti-cancer efficacy too.

Methodology: Plant extracts were noted to be non-toxic to normal breast epithelial cells but toxic to breast cancer cells. However, the ethyl acetate extract of the Lycopersicon esculentum fruits was most effective amongst all other extracts.

Results: Data suggest that Lycopersicon esculentum fruits potentiate DNA damage mediated apoptosis and negatively regulate cell proliferation, angiogenesis and metastasis in vitro and in vivo. Moreover, the anti-cancer phenomenon was found to be the induction of inflammatory cytokines mediated oxidative stress in breast cancer.

Conclusion: Lycopersicon esculentum fruits and Cassia fistula pods show excellent cancer therapeutics to induce DNA damage, inhibit angiogenesis and metastasis simultaneously.

Keywords: Lycopersicon esculentum fruits, Cassia fistula pods, Breast cancer, Angiogenesis, Apoptosis, MCF-10A

\section{INTRODUCTION}

Breast cancer is the most common reason for cancer mortality in women. It is a hormonal malignancy that is influenced by several hormones and growth factors. ${ }^{1,2}$ It is predicted that one in every eight females in the world will develop breast cancer and only $5-10 \%$ of cases of this cancer are associated with genetic disorders, whereas the remaining 90-95\% of cases are linked to environmental and lifestyle factors. ${ }^{3,4}$ Breast cancer represents 1 in 4 cancers diagnosed among women globally. ${ }^{5,6}$ Modern cancer therapeutics utilizes the implications of natural products like Vinca rosea, Allium sativum, Viscum album, Zingiber officinale, Taxus wallichiana, Panax pseudoginseng, Tinospora cordifolia. ${ }^{7}$ In addition, fruits of Lycopersicon esculentum (tomato), pods of Cassia fistula (also known as Garmalo of the family: Fabaceae) are natural bioactive compounds and do not express toxic effects in our body.

Lycopersicon esculentum (tomato), of the family Solanaceae, is one the most widely used fruit. A diet that includes tomato and tomato-based products reduces the risk of pancreatic cancer ${ }^{8}$ and also some chronic diseases like cancer and cardiovascular disease. Carotenoids are found to restrain the growth of many cancer lines like prostate, mammary, lung, colon, leukaemia. ${ }^{9-13}$

Cassia fistula (also known as Garmalo of the family: Fabaceae) is a potent anticancer agent on human colon cancer. ${ }^{15}$ It is reported to possess hepatoprotective, anti-inflammatory, antitussive, antifungal and antibacterial. Cassia fistula bark extract taken orally on DMBA painted animals completely prevent the formation of oral squamous cell carcinoma. ${ }^{16,17}$

\section{Corresponding Author:}

Mr. Ajmire Prashant, School of Pharmacy, Dr. A. P. J. Abdul Kalam University, Indore (MP), India.

Contact: +917588962187; Email: prashantajmire@gmail.com

ISSN: $2231-2196$ (Print)

ISSN: 0975-5241 (Online)

Received: 01.01 .2021

Revised: 15.02 .2021

Accepted: 22.03 .2021

Published: 26.05 .2021 
One of the components of this flower, Rhein is anticarcinogenic. ${ }^{15}$ Also, Cassia fistula possess antiestrogenic activity. ${ }^{18}$ Lupeol, one of its components is proven to be an antitumor agent. ${ }^{16}$

In our present work, we have comparatively analyzed the in vitro anticancer efficacy of different extracts of Lycopersicon esculentum fruits and Cassia fistula pods by using a human breast cancer cell line. We have also provided the mechanistic activity behind their anti-cancer activity and further, we have validated the in vitro result by our developed mice xenograft model system.

\section{MATERIALS AND METHODS}

\section{Cell culture and chemicals:}

All the cell culture chemicals were procured from GIBCO, ThermoFisher Scientific, India. Antibodies were purchased from Elabscience Biotechnology Inc. 3-(4,5-dimethylthiazol-2yl)-2,5-diphenyl tetrazolium bromide (MTT) used for this study were purchased from Sigma Chemicals (Sigma, St Louis, MO, USA).

\section{Cell proliferation and viability assay:}

The short-term anchorage-dependent cell viability of different plant extract treated both the normal and breast cancer cells was measured by performing the MTT assay. ${ }^{19}$ Experiment was performed according to methods published recently. Experiments were carried out three times and each data point was calculated in a triplicate manner.

\section{Measurement of DNA damage by alkaline single cell gel electrophoresis (SCGE) or Comet assay:}

To measure the DNA damaging ability of the plant extracts in the breast cancer cells, single-cell gel electrophoresis or comet assay was performed according to manufactures protocol. ${ }^{20}$

\section{vH2AX Immunofluorescence assay:}

$\gamma \mathrm{H} 2 \mathrm{AX}$ immunofluorescence assay was performed to check the DNA damaging abilities of the plant extracts in breast cancer. ${ }^{20}$ MCF-7 cells were grown on a 96-well tissue culture plate and treated with the plant extract for indicated time duration. Then the cells were washed with 1X PBS and fixed with acetone: methanol $(1: 1)$ for 20 mins at $-20^{\circ} \mathrm{C}$. Cells were washed twice with $1 \mathrm{X}$ PBS and incubated with primary antibody anti- $\gamma \mathrm{H} 2 \mathrm{AX}$ (1:500 dilutions in 1X PBS) for $2 \mathrm{~h}$ at $37^{\circ} \mathrm{C}$. Then unbound antibodies were removed by washing twice with $1 \mathrm{X}$ PBS and incubated with secondary antibody conjugated with TRITC (1:750 dilutions in 1X PBS) for $1 \mathrm{~h}$ at $37^{\circ} \mathrm{C}$. Cells were washed thrice with $1 \mathrm{X}$ PBS and nuclei were counter-stained with DAPI. Finally, the images were captured using an inverted fluorescence microscope (Nikon, Japan) at 20X magnification.

\section{DAPI nuclear staining:}

To determine the DNA damage mediated apoptosis of breast cancer cells after treatment with the indicated plant extract, 4',6-diamidino-2-phenylindole (DAPI) nuclear staining was carried out. The experiment was carried according to the methods given in the article..$^{21}$

\section{Matrigel invasion assay:}

The matrigel invasion assay was performed to evaluate the effectiveness of the plant extracts to inhibit the invasive properties of the breast cancer cells. Briefly, $3 \times 10^{5}$ cells were resuspended in $100 \mu 1$ of serum-free media and seeded either on uncoated inserts (migration) or on the matrigel coated inserts (invasion). Complete media was added to the lower chamber and incubated for $24 \mathrm{~h}$. Non-invaded cells were removed and finally invaded cells were fixed with $4 \%$ paraformaldehyde, stained with DAPI and counted at 40X under the fluorescence microscope. The data were expressed as \% invasion against cell types. \% invasion was calculated using the following formula:

$\%$ Invasion $=[$ Number of cells invaded the matrigel coated inserts towards the complete media / Number of cells migrated the uncoated inserts towards the complete media] $\times 100$.

\section{Western blot analysis:}

Western blot analysis was performed to check the expressions of several apoptotic, anti-apoptotic and angiogenic markers before and after the exposure of the plant extracts on MCF-7 cells. The assay was performed according to methods given by Chatterjee ${ }^{20}$

\section{ELISA (Enzyme-linked Immunosorbent Assay)}

Expressions of proliferation marker, Ki-67 and other prime angiogenic markers (like VEGF-A, ANG-1, ANG-2) were measured by ELISA. It was performed according to manufactures protocol and the final result was expressed in Optical density. ${ }^{20}$

Annexin V-FITC/PI dual staining for apoptosis measurement:

Annexin-V-FITC/PI dual staining was carried out to validate the apoptotic potentiality of the plant extracts in breast cancer cells. ${ }^{21}$ Briefly, 8000-10,000 cells/well were seeded in 96 -well plate and after $24 \mathrm{~h}$ of the incubation, treatment procedure was followed as mentioned earlier. Then, the cells were harvested and washed twice with 1X PBS. After that, the cells were resuspended in $1 \mathrm{X}$ binding buffer and stained with Annexin-V-FITC and PI detection kit (Sigma) according to the protocol provided by the manufacturer. The stained 
cells were incubated for 10 min and analyzed using a FACS Canto-II flow cytometer (BD, USA).

\section{Determination of oxidative stress, enzymatic antioxidant and catalase activity:}

After the above-mentioned exposures of the plant extracts in MCF-7 cells, the Reduced Glutathione (GSH) level was measured by commercially available ELISA kits according to the manufacturer's instructions (Cayman, Ann Arbor, MI). Also, the level of Superoxide Dismutase (SOD) was measured according to the manufacturer's instructions (Cayman, Ann Arbor, MI) as markers of enzymatic antioxidant activity. In addition to this, catalase and malondialdehyde (MDA) levels were also measured according to the kit method.

\section{Establishment of mice xenograft model sys- tem:}

Mice xenograft model was developed to check the anti-cancer efficacy of the plant extracts in vivo model system. ${ }^{22} 6$ weeks old female nude mice were housed in a proper light/ dark cycle of 12/12 h. All the experiments were approved by the Institutional Animal Ethical Committee. $1 \times 10^{7}$ MCF-7 cells in $200 \mu 1$ freshly prepared sterile 1 X PBS were injected into the left mammary fat pads of 3 different groups of mice (each group contained 5 mice). The first group of mice were used as control and the other 2 groups of mice were implanted with MCF-7 cells for tumour development. Tumour growth and mice health were monitored regularly. After 25 days of implantation of cells when a measurable amount of tumour was noticed, then $100 \mu 1$ of the plant extracts (at their $\mathrm{IC}_{50}$ concentrations of in vitro system) was administered in 2 groups by oral route. Mice body weight was measured every alternative day for 55 days. Tumour dimensions were measured using slide callipers, and tumour volumes were calculated using the formula: $\left(\mathrm{W}^{2} \times \mathrm{L}\right) / 2$, where width $(\mathrm{W}) \leq$ length (L). Then, the mice were euthanized and tumour tissues were collected and processed for H\&E staining and immunohistochemistry (IHC) analysis.

\section{Histopathological study (H\&E staining) analysis of mice tissue:}

Histopathological staining was performed to validate the establishment of the mice xenograft model..$^{22}$ For this, paraffinembedded mice breast tissues were cut into a section of $5 \mu \mathrm{m}$ thickness, mounted on slides and heated at $60^{\circ} \mathrm{C}$ for $30-40$ min. Tissue sections were deparaffinized with xylene and rehydrated with immersing in decreasing concentrations of alcohols (100\%, 90\%, 70\% and 50\%, respectively). Then the slides were dipped into hematoxylin followed by eosin stain (H\&E) and rinsed in distilled water. Then slides were subjected to dehydration by dipping in increasing concentrations of alcohols $(50 \%, 70 \%, 90 \%$ and $100 \%)$ and finally incubated in xylene for 2 mins. The sections were mounted by using the DPX mountant. Images were captured in a bright-field microscope (Leica DM200, USA) at 20X magnification.

\section{Immunohistochemistry (IHC) analysis of xeno- graft mice tissue:}

Immunohistochemistry analysis of the breast tissues of the mice was carried out to check the expressions of angiogenic and metastatic markers before and after the exposure of the plant extracts. For this, tissue specimens were dewaxed with xylene, rehydrated with decreasing concentrations of ethanol $(100 \%, 90 \%$, and $70 \%$, respectively).IHC has performed accounting to methods published recently. ${ }^{22}$

\section{Statistical analysis}

Statistical analysis was performed using GraphPad Prism 5.0 software, USA. Results represented here were the mean \pm standard deviation (SD) of three separate experiments. Data were analyzed using one-way and two-way ANOVA where applicable, followed by Bonferroni's multiple comparison test. Statistical significance of the difference in the central tendencies compared to control groups were designated as '*’' $(\mathrm{p}<0.05),{ }^{\prime * *}(\mathrm{p}<0.001)$ and '***' $(\mathrm{p}<0.0001)$.

\section{RESULTS AND DISCUSSION:}

Different extracts of Lycopersicon esculentum fruits and Cassia fistula pods inhibit the breast cancer cells viability:

In our current piece of work, no notable decrease in cell viability was noticed in all the treatment conditions of Lycopersicon esculentum fruits and Cassia fistula pods on noncancerous MCF-10A cells at different times points $(24 \mathrm{~h}, 48 \mathrm{~h}$ and $72 \mathrm{~h}$ ) (Figure 1A, B). Then, the effects of the Lycopersicon esculentum fruits at various concentrations $(10-1000 \mu \mathrm{g} /$ $\mathrm{ml})$ and at various time points ( $24 \mathrm{~h}, 48 \mathrm{~h}$ and $72 \mathrm{~h}$ ) revealed EAELE to be most potent than MELE, AELE and BELE in inhibiting proliferation of MCF-7 human breast cancer cell line (Figure 2A). After exposure of different extracts of Cassia fistula pods in MCF-7 cell line, at various concentrations (i.e. 10, 100, 200, 300, 500 and $1000 \mu \mathrm{g} / \mathrm{ml}$ ) for different time point ( $24 \mathrm{~h}, 48 \mathrm{~h}$ and $72 \mathrm{~h}$ ), the anti-proliferative order was found to be AECF $>$ MECF $>$ BECF $>$ EAECF. (Figure 2B). The time-dependent cell viability of MCF-7 after the exposure of all the different plant extracts was documented in Table 1.

Further, the anti-cell proliferative abilities of the abovementioned plant extracts were checked by evaluating the expression of Ki-67 in the MCF-7 cell line (Figure 2C). Approximately, 1.5 and 1.4-fold decrease in Ki-67 expression was noted upon the $24 \mathrm{~h}$ exposure of EAELE and AECF, respectively (Figure 2C). 
Lycopersicon esculentum fruits and Cassia fistula pods caused DNA damage in the MCF-7 breast cancer cell line:

Figure $3 \mathrm{~A}$ shows the comet tail formation in EAELE and AECF treated MCF-7 cell lines. Significant comet tails were formed in MCF-7 cells after the exposure of EAELE and AECF (Figure 3B). To confirm that $\gamma-\mathrm{H} 2 \mathrm{AX}$ immunofluorescence assay was performed (Figure $3 \mathrm{C}$ ). $\gamma-\mathrm{H} 2 \mathrm{AX}$ is the phosphorylated form of $\mathrm{H} 2 \mathrm{AX}$ and it expresses when DNA damage has occurred. Significant expression of $\gamma$-H2AX was noted in plant extracts treated MCF-7 as compared with untreated control (Figure 3C, D).

Lycopersicon esculentum fruits and Cassia fistula pods caused apoptosis mediated cell death of MCF-7 breast cancer cells:

The above result of EAELE and AECF suggested that the plant extracts caused significant DNA damage in breast cancer cells. Again, to validate this, DAPI nuclear staining was carried out to check the phenotypical microscopic changes of the nucleus upon the exposure of those plant extracts. Upon the exposure of EAELE and AECF, significant toxicity in the nucleus was observed (Figure 4A, B). Next, we wanted to check the apoptotic potentiality of the plant extracts and for this, we have checked the expressions of some representative apoptotic and anti-apoptotic proteins by western blot analysis. Figure 4C shows the expressions of BCL-XL (anti-apoptotic marker), BAX (apoptotic marker) and CASPASE-3 after the exposure of EAELE and AECF in the MCF-7 cell line. Higher expressions of BAX and CASPASE-3 and lower expression of BCL-XL were found after the treatment of EAELE and AECF (Figure 4C). Approximately, 2.5-fold downregulation, 2.7- and 3.4-fold upregulation of BCL-XL, BAX and CASPASE-3 were recorded in EAELE treated MCF-7, respectively (Figure 4C). When we treated the cells with AECF, approximately, 1.66-fold decreased, 2.1- and 2.9-fold enhanced expression of BCL-XL, BAX and CASPASE-3 were found, respectively (Figure 4C). GAPDH served as loading control in all the treatment conditions.

Again, to validate this, Annexin-V-FITC/ PI staining was performed after treating the cells with the plant extract and was analyzed by flow cytometry. Figure 4D showed the population of cells undergoing early apoptosis, late apoptosis or necrosis in untreated MCF-7 (control), EAELE and AECF treated cells, respectively. Comparatively more cell death [early apoptosis (Q4) + late apoptosis (Q2) + necrosis (Q1)] was noted in treated breast cancer cells as compared with untreated control. Approximately, 27\% of cells were undergone cell death upon EAELE treatment; wherein the AECF exposed cells, the value was found to be $22 \%$ (Figure 4E).

Lycopersicon esculentum fruits and Cassia fistula pods inhibit angiogenesis and metastasis in MCF-7 breast cancer cells:
At the very first, expressions of prime angiogenic markers like VEGF-A, TGF- $\beta$, MMP-9 and MMP- 2 were checked by western blot analysis. Figure 5A showed the expression of VEGF-A, TGF- $\beta$, MMP-9 and MMP-2 in EAELE and AECF treated MCF-7 cells. In EAELE and AECF treated cells, 2.5, 2.5 and 2, a 2-fold decrease in expression of VEGF-A and TGF- $\beta$ were measured, respectively. In addition, expressions of MMP-9 and MMP-2 were found to be decreased by 2.5, 1.67 and 1.67, 1.4-fold, respectively (Figure 5A).

Further, secretory angiogenic markers like Ang-1 (Angiopoietin-1), Ang-2 and VEGF-A were checked in the supernatant of MCF-7 and the treatment condition of EAELE and AECF (Figure 5B, C, D). Here also, decreased expressions of Ang1, Ang-2 and VEGF-A were observed in EAELE and AECF treatment conditions. However, EAELE treatment was found to be more effective than that of AECF in all the above cases.

Next, the involvement of these plant extracts on metastasis was analyzed by matrigel invasion assay and by checking the expressions of some prime metastasis markers (CD-44, CD-133, OCT-4) by Western Blot analysis. Figure 5E shows the percentage of cells invaded in the matrigel, which is indirectly proportional to the anti-invasive potentiality of our plant extracts. From Figure 5E it was found that EAELE was effective to inhibit the invasiveness of breast cancer cells than that of AECF. Western Blot analysis showed a similar observation that downregulation of CD-44, CD133 and OCT- 4 was observed in the treatment condition of EAELE and AECF. GAPDH served as a loading control (Figure 5F).

Lycopersicon esculentum fruits and Cassia fistula pods mediated anti-cancer activity may be through induction of oxidative stress of breast cancer cells:

Upon the treatment of EAELE and AECF, SOD level was found to be down-regulated (Figure 6A). EAGLE and AECF induced oxidative stress was further evidenced by depletion of catalase activity in breast cancer cells. Upon the treatment with EAELE and AECF, catalase level was found to be inhibited (Figure 6B). To validate the above observation, we checked the MDA level in different treatment conditions of EAELE and AECF in breast cancer cells. Elevation of lipid peroxides was noted upon the treatment with both the plant extracts (Figure 6C). Finally, GSH level was also assayed and depletion of GSH upon the treatment with EAELE and AECF, further supported the plant extracts mediated oxidative stress (Figure 6D). In all the cases EAELE was found to be more effective than AECF.

Further, to illustrate the mechanism behind the plant extracts mediated oxidative stress, the expressions of some inflammatory and anti-inflammatory cytokines were checked upon the treatment of plant extracts in MCF-7 cells (Figure 6). 
Lycopersicon esculentum fruits and Cassia fistula pods exhibit anti-cancer potentiality in in-vivo mice xenograft model system

Our cell culture-based study revealed the anti-cancer efficacy of the above-mentioned different plant extracts in vitro model system. Further to validate this, and in vivo mice, a xenograft model was developed by using nude mice. After 10 days of implantation of MCF-7 cells, tumour formation was noted in the mice. The body weight of the mice was found to be significantly decreased and the tumour volume was increased day-by-day (Figure 7A, B). After 25 days of administration of cells, tumour volume was found to be significantly increased and the bodyweight of mice was noted to be significantly decreased (Figure 7A, B). Oral administration of the plant extracts was given for the next 25 days on every alternative day. Interestingly, little recovery from lost body weight was noted and reduction of tumour volume was also noticed at the end of the treatment (Figure 7A, B). After 50 days of administration of cells, the mice were euthanized and tumour tissues were studied for H\&E staining as well as IHC. H\&E staining showed enlarged and non-uniform nuclei in tumour bearing mice in comparison to control mice (Figure 7C).

Then, to evaluate the impact of the plant extracts in vivo model system, the expressions of representative proteins of metastasis and angiogenesis were analyzed using the IHC of the tissue section. A higher expression of VEGF-A and OCT-4 breast tissues were noted in tumour-bearing mice. But EAELE and AECF treatment significantly decreased the above-elevated levels of markers in tissue samples (Figure 7D).

\section{DISCUSSION}

In order to illustrate the mechanism of these plant extracts mediated anti-cancer efficacy, we first checked their effect in normal epithelial cells. MCF-10A is a well-established model for normal breast epithelial cells. In our current piece of work, when we exposed the MCF-10A cells to different extracts (aqueous, methanol, butanol and ethyl acetate) of Lycopersicon esculentum fruits (AELE; MELE; BELE and EAELE) and Cassia fistula pods (AECF; MECF; BECF and EAECF) for 24,48 and $72 \mathrm{~h}$, cell proliferation was found to be not significantly affected. Fifty percent decrease in cell viability $\left(\mathrm{IC}_{50}\right.$ value) was not observed in all the treatment conditions of Lycopersicon esculentum or Cassia fistula. This signifies that the plant extracts are non-toxic to normal cells.

Next, to evaluate the effectiveness of the plant extracts in inhibition of cell viability, ER-positive MCF-7 cells were treated with the above-mentioned extracts of Lycopersicon esculentum and Cassia fistula. MTT cell viability data showed the $\mathrm{IC}_{50}$ value of all the extracts at their different time point treatment. However, ethyl acetate extract of $L y$ copersicon esculentum fruits (EAELE) and aqueous extract of Cassia fistula pods (AECF) was noted as the best possible experimental conditions in the MCF-7 cell line. Taken together, EAELE and AECF showed anti-cell viability efficacy in the MCF-7 cell line and hence, in all the rest set of experiments, we have used EAELE and AECF at their $\mathrm{IC}_{50}$ value (24h). Again, to support the plant extracts mediated decrease in cell viability, $\mathrm{Ki}-67$ expression was checked by indirect ELISA. Decreased expression of Ki-67 was noted in all the treatment conditions of plant extracts in MCF-7. Moreover, the lowest expression of $\mathrm{Ki}-67$ was noted in EAELE treated cells, which further suggested the more anti-cell proliferative potentiality of EAELE than AECF.

Further to validate the in vitro data, we have checked the effectiveness of these plant extracts in vivo model system. For this, we have developed a mice xenograft model system by injecting MCF-7 breast cancer cells into the breast fat pad of female nude mice. 2 days after injecting the cells, when visible tumour formed, different groups of mice were treated with EAELE and AECF. After 55 days, mice were euthanized and tissues were examined for H\&E staining or IHC. A decrease in increased tumour volume and an increase in reduced body weight suggested the development of a tumour model. Again, H\&E data proved the true development of tumours in the mice xenograft model system. Immunohistochemical analysis of angiogenic marker VEGF-A and metastatic marker OCT-4 showed that treatment with EAELE and AECF reduced tumour-induced angiogenesis and metastasis.

\section{CONCLUSION}

By using the MCF-7 breast cancer model system, we have successfully evaluated the anti-cancer potentiality of $\mathrm{Lyco}$ persicon esculentum and Cassia fistula. Taking all the data together, the present piece of work seems to be an excellent comparative and mechanistic study to evaluate the anti-cancer activity of Lycopersicon esculentum and Cassia fistula in breast cancer. Ethyl acetate extract of Lycopersicon esculentum fruit and aqueous extract of Cassia fistula pod was found to be more potent than the other extracts in breast cancer.

\section{ACKNOWLEDGEMENTS}

Authors acknowledge the support provided by Dr. Rajendra Gode Institute of Pharmacy, Amravati, Maharashtra, India for the present work

Conflict of interest: The authors declare that they have no conflict interests

Funding: NIL 


\section{Author contribution:}

Ajmire Prashant: Concept, methodology, analysis of data, wrote the first draft.

Patel Rakesh: Concept, methodology, wrote first draft, supervision.

Kayande Nandu: analysis of data, wrote the first draft, edited draft

Bakal Ravindra: revised manuscript, provided resources

\section{REFERENCES}

1. Topper YJ, Sankaran L, Chomczynski P, Prosser C, Qasba P. Three stages of responsiveness to hormones in the mammary cell. Ann N Y Acad Sci. 1986;4641-10.

2. Russo J, Tahin Q, Lareef MH, Hu Y-F, Russo IH. Neoplastic transformation of human breast epithelial cells by estrogens and chemical carcinogens. Environ Mol Mutagen. 2002;39(23):254-263.

3. van Geel AN, Lans TE, Haen R, Tjong Joe Wai R, MenkePluijmers MBE. Partial Mastectomy and M. Latissimus Dorsi Reconstruction for Radiation-induced Fibrosis After Breastconserving Cancer Therapy. World J Surg. 2011;35(3):568-572.

4. Henríquez-Hernández LA, Carmona-Vigo R, Pinar B. Combined low initial DNA damage and high radiation-induced apoptosis confers clinical resistance to long-term toxicity in breast cancer patients treated with high-dose radiotherapy. Radiat Oncol. 2011;6(1):60.

5. Mitri Z, Constantine T, O'Regan R. The HER2 Receptor in Breast Cancer: Pathophysiology, Clinical Use, and New Advances in Therapy. Chemother Res Pract. 2012;2012743193.

6. Bello E, Taraboletti G, Colella G. The Tyrosine Kinase Inhibitor E-3810 Combined with Paclitaxel Inhibits the Growth of Advanced-Stage Triple-Negative Breast Cancer Xenografts. Mol Cancer Ther. 2013;12(2):131-140.

7. Hsia T-C, Tu C-Y, Chen Y-J. Lapatinib-Mediated Cyclooxygenase-2 Expression via Epidermal Growth Factor Receptor/HuR Interaction Enhances the Aggressiveness of Triple-Negative Breast Cancer Cells. Mol Pharmacol. 2013;83(4):857-869.

8. Bhowmik D, Paswan S, Srivastava S. Tomato-A Natural Medicine and Its Health Benefits. J Pharmacogn Phytochem. 2012;133-43.

9. Stajčić S, Ćetković G, Čanadanović-Brunet J, Djilas S, Mandić A, Četojević-Simin D. Tomato waste: Carotenoids content, antioxidant and cell growth activities. Food Chem. 2015;172225232.
10. Giovannucci E. Tomatoes, Tomato-Based Products, Lycopene, and Cancer: Review of the Epidemiologic Literature. JNCI J Natl Cancer Inst. 1999;91(4):317-331.

11. Giovannucci E. A Review of Epidemiologic Studies of Tomatoes, Lycopene, and Prostate Cancer. Exp Biol Med. 2002;227(10):852-859.

12. Palozza P, Simone RE, Catalano A, Mele MC. Tomato Lycopene and Lung Cancer Prevention: From Experimental to Human Studies. Cancers (Basel). 2011;3(2):2333-2357.

13. Yang T, Yang X, Wang X, Wang Y, Song Z. The role of tomato products and lycopene in the prevention of gastric cancer: A meta-analysis of epidemiologic studies. Med Hypotheses. 2013;80(4):383-388.

14. Karimi G, Ramezani M, Abdi A. Protective effects of lycopene and tomato extract against doxorubicin-induced cardiotoxicity. Phytother Res. 2005;19(10):912-914.

15. Duraipandiyan V, Baskar AA, Ignacimuthu S, Muthukumar C, Al-Harbi NA. Anticancer activity of Rhein isolated from Cassia fistula L. flower. Asian Pacific J Trop Dis. 2012;2S517-S523.

16. Danish M, Singh P, Mishra G, Srivastava S, Jha KK, Khosa RL. Cassia fistula Linn. (Amulthus)- An Important Medicinal Plant: A Review of Its Traditional Uses, Phytochemistry and Pharmacological Properties. J Nat Prod Plant Resour. 2011;1101-118.

17. Vasudevan K, Manoharan S, Alias LM, Balakrishnan S, Vellaichamy L GM. Evaluation of Chemopreventive Efficacy of Cassia fistula in 7, 12 Dimethyl Benz (A) Anthracene (DMBA) induced Oral Carcinogenesis. Int J Chem Sci. 2008;1(1):101-118.

18. Yadav R JG. Effect of petroleum ether extract of Cassia fistula seeds on uterine histoarchitecture of ovariectomized female rats. Indian J Fundam Appl Life Sci. 2013;3(1):167-174.

19. Chatterjee S, Sinha S, Molla S, Hembram KC, Kundu CN. PARP Inhibitor Veliparib (ABT-888) enhances the anti-angiogenic potentiality of Curcumin through deregulation of NECTIN-4 in oral cancer: Role of nitric oxide (NO). Cell Signal 2021;80109902.

20. Chatterjee $\mathrm{S}$, Kundu CN. Nanoformulated quinacrine regulates NECTIN-4 domain-specific functions in cervical cancer stem cells. Eur J Pharmacol. 2020;883173308.

21. Nayak D, Tripathi N, Kathuria D. Quinacrine and curcumin synergistically increased the breast cancer stem cells death by inhibiting ABCG2 and modulating DNA damage repair pathway. Int J Biochem Cell Biol 2020;119105682.

22. Hembram KC, Chatterjee S, Sethy C. Comparative and Mechanistic Study on the Anticancer Activity of Quinacrine-Based Silver and Gold Hybrid Nanoparticles in Head and Neck Cancer. Mol Pharm. 2019;16(7):3011-3023. 

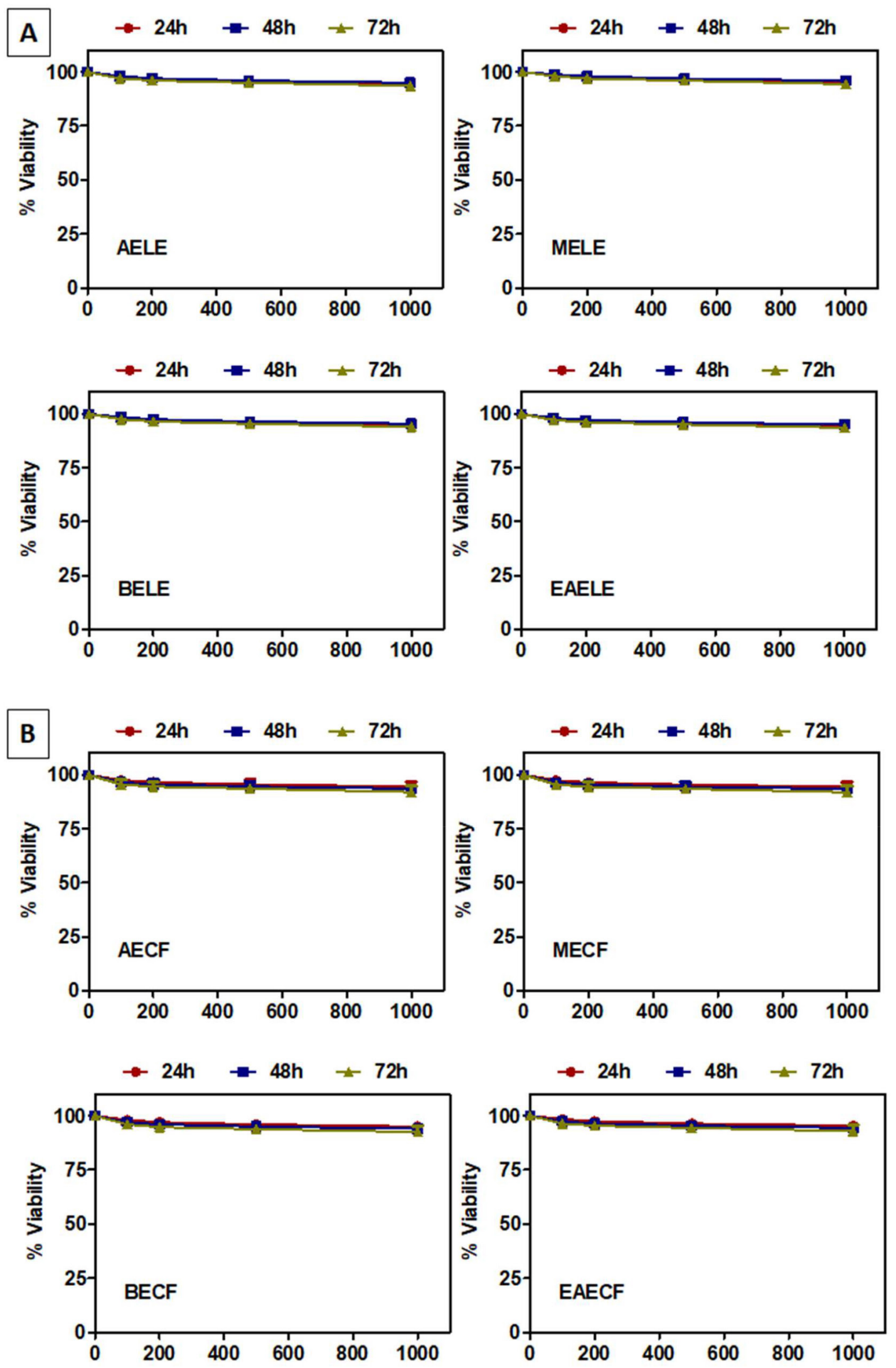

Figure 1: Effect of various extracts of Lycopersicon esculentum fruits $(A)$ and Cassia fistula pods (B) on cell viability of MCF-10A cell line at different time points $(24 \mathrm{~h}, 48 \mathrm{~h}$ and $72 \mathrm{~h})$. Values were represented as Mean \pm SD of 3 independent experiments. 

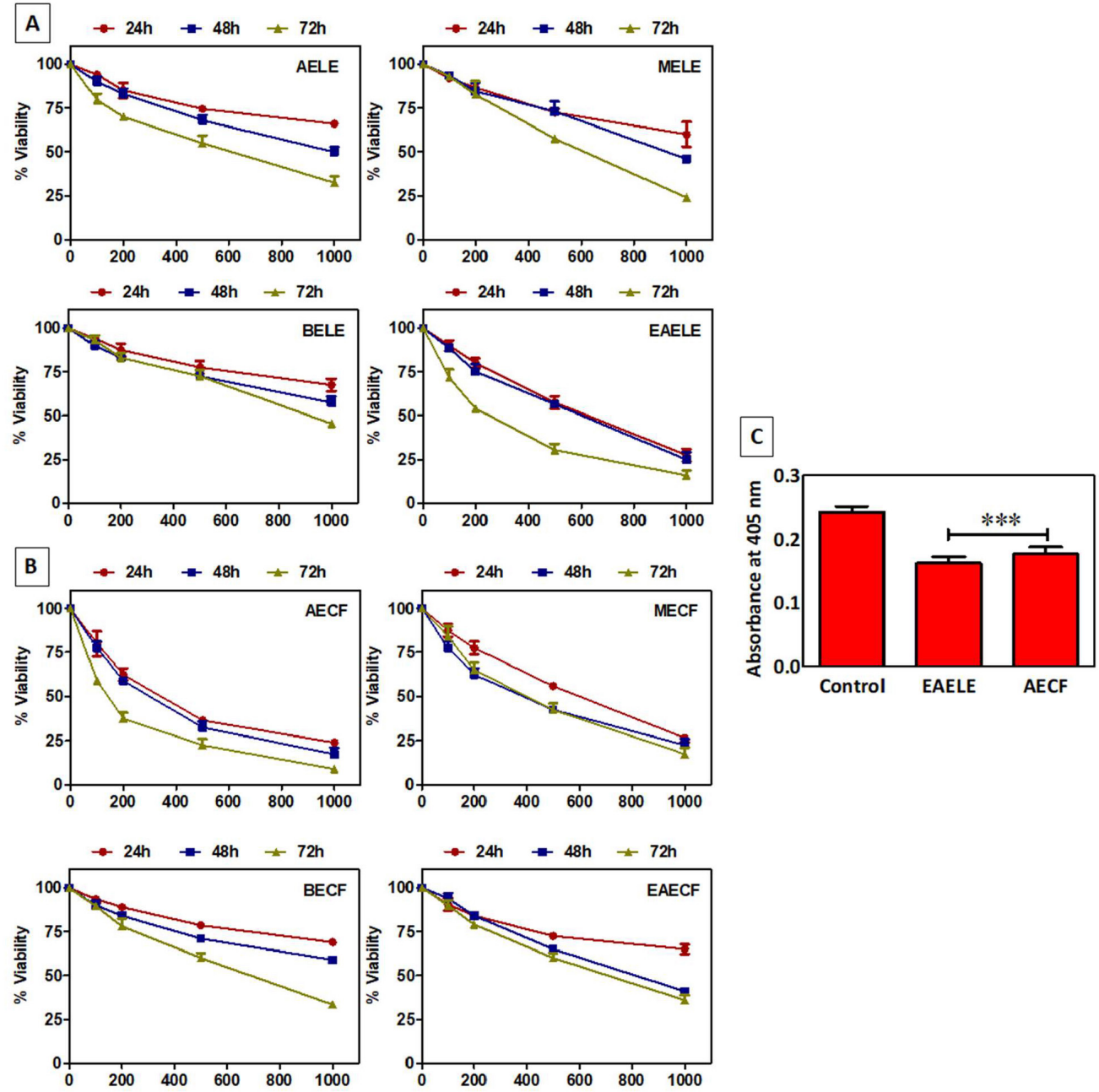

Figure 2: Effect of various extracts of Lycopersicon esculentum fruits (A) and Cassia fistula pods (B) on cell viability and cell proliferation of MCF-7 breast cancer cell line. (C) Expression of Ki-67 in MCF-7 human breast cancer cell line after the exposure of EAELE and AECF. Values were represented as Mean \pm SD of 3 independent experiments.

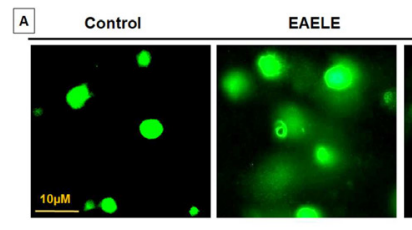

C DAPI YH2AX TRITC MERGE

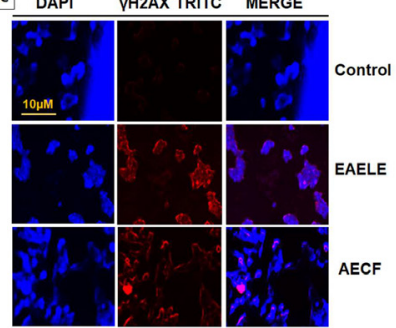

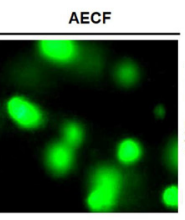

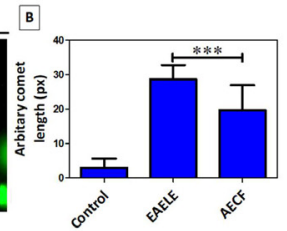

D

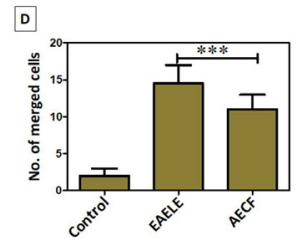

Figure 3: Lycopersicon esculentum fruits and Cassia fistula pods caused DNA damage in MCF-7 cells. (A) Comet assay was performed after the exposure of EAELE and AECF in MCF-7 cells. (B) Graphical representation of comet tail length. (C) Expression of $\mathrm{y}-\mathrm{H} 2 \mathrm{AX}$ upon the treatment of EAELE and AECF. (D) Graphical representation of Figure 4C. Experiments were carried out three times and a representative image was provided. 


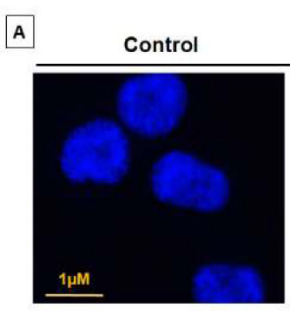

D

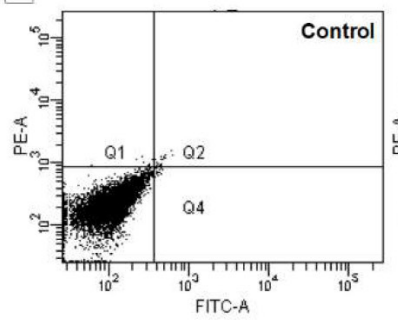

EAELE
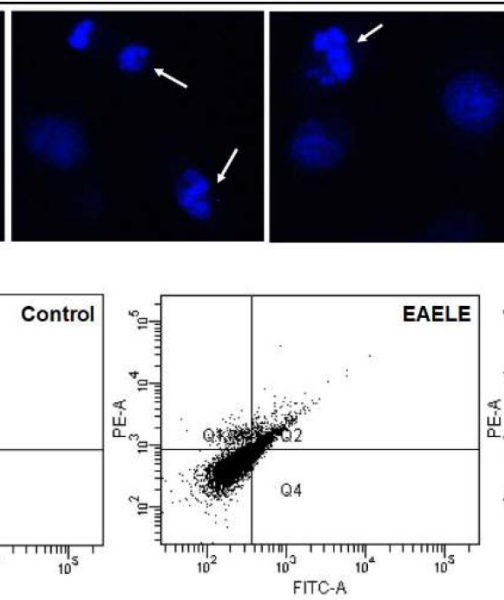

B

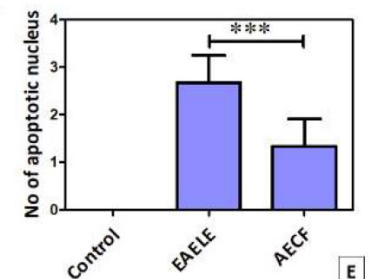

c

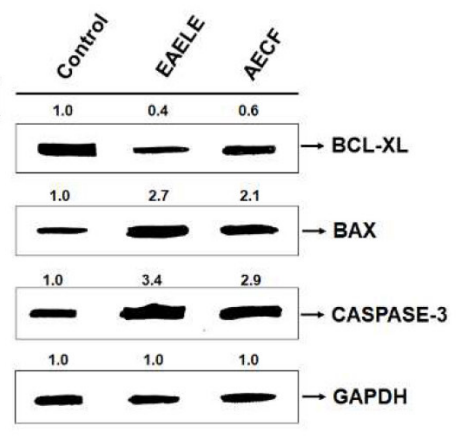

Figure 4: Lycopersicon esculentum fruits and Cassia fistula pods caused apoptosis in MCF-7 cells. (A) DAPI nuclear staining after the exposure of EAELE and AECF in MCF-7 cells. (B) Graphical representation of Figure 5A. (C) Expression of BCL-XL, BAX, CASPASE-3 upon the treatment of the plant extracts. GAPDH served as a loading control. (D) Annexin-V-FITC analysis of cell death population after treating MCF-7 cells with EAELE and AECF. (E) Graphical representation of Figure 5D. Experiments were carried out three times and representative data was provided.
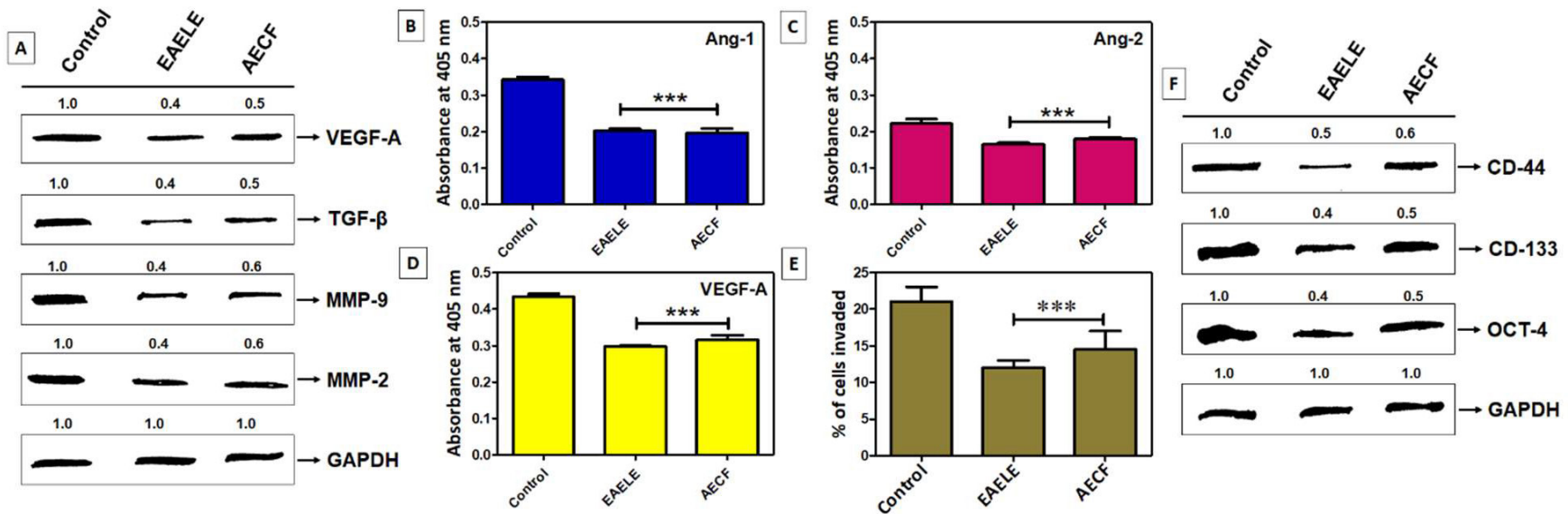

Figure 5: Lycopersicon esculentum fruits and Cassia fistula pods caused inhibition of angiogenesis and metastasis in MCF-7 cells. (A) Western blot analysis of prime angiogenic proteins. GAPDH served as a loading control. (B), (C) and (D) Expression of ANG-1, ANG-2 and VEGF-A in the supernatant of MCF-7 cells upon the treatment of EAELE and AECF. (E) Anti-invasiveness potentiality of EAELE and AECF in MCF-7 cells. (F) Expressions of prime metastatic markers after the exposure of EAELE and AECF in MCF-7 cells. Experiments were carried out three times and representative data was provided. 

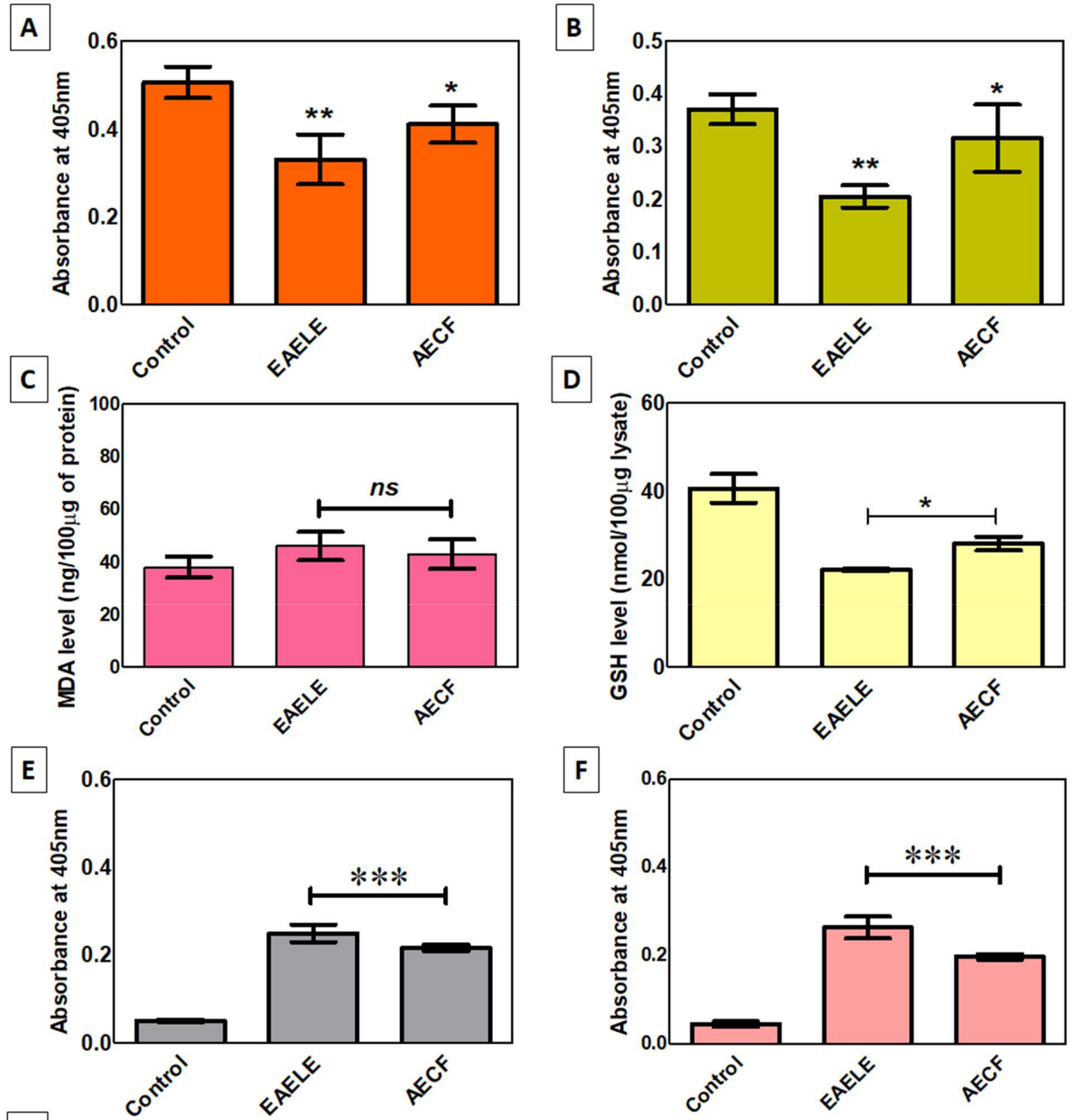

$\mathbf{G}$
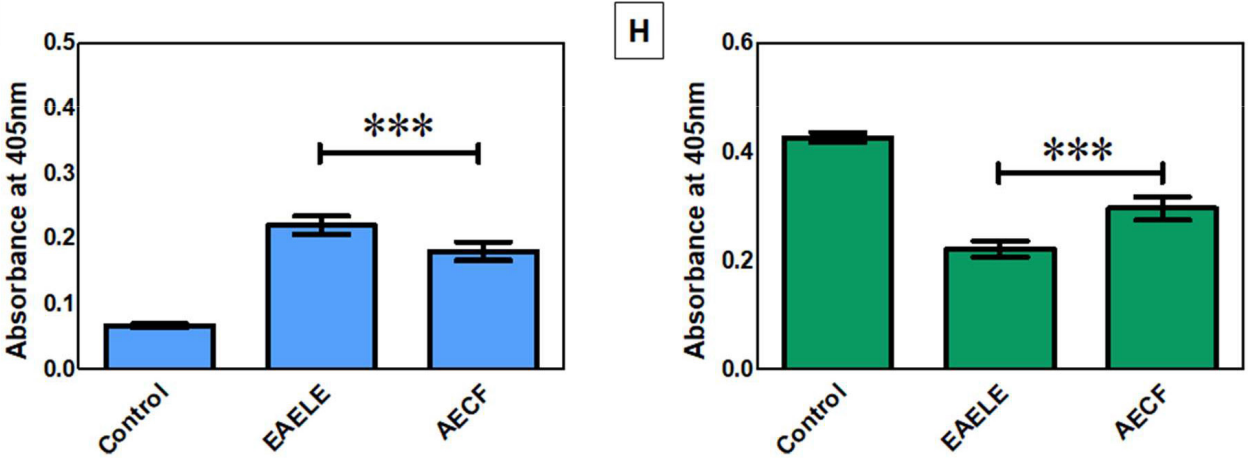

Figure 6: Lycopersicon esculentum fruits and Cassia fistula pods caused induction of oxidative stress in MCF-7 cells. (A) Biochemical detection of Superoxide dismutase, Levels of Catalase (B), lipid peroxides (C) and GSH (D) MCF-7 cells after the treatment of EAELE and AECF in MCF-7 breast cancer cells. $(E)$ to $(\mathrm{H})$ Effect of EAELE and AECF on pro and anti-inflammatory cytokines in MCF-7 cells. (E-H) are the graphical representation of the optical density of cell supernatants at $405 \mathrm{~nm}$ for TNF-a, IL-1 $1 \beta$, IL-6 and IL-10. Data represent mean \pm S.D. of 3 independent experiments. 


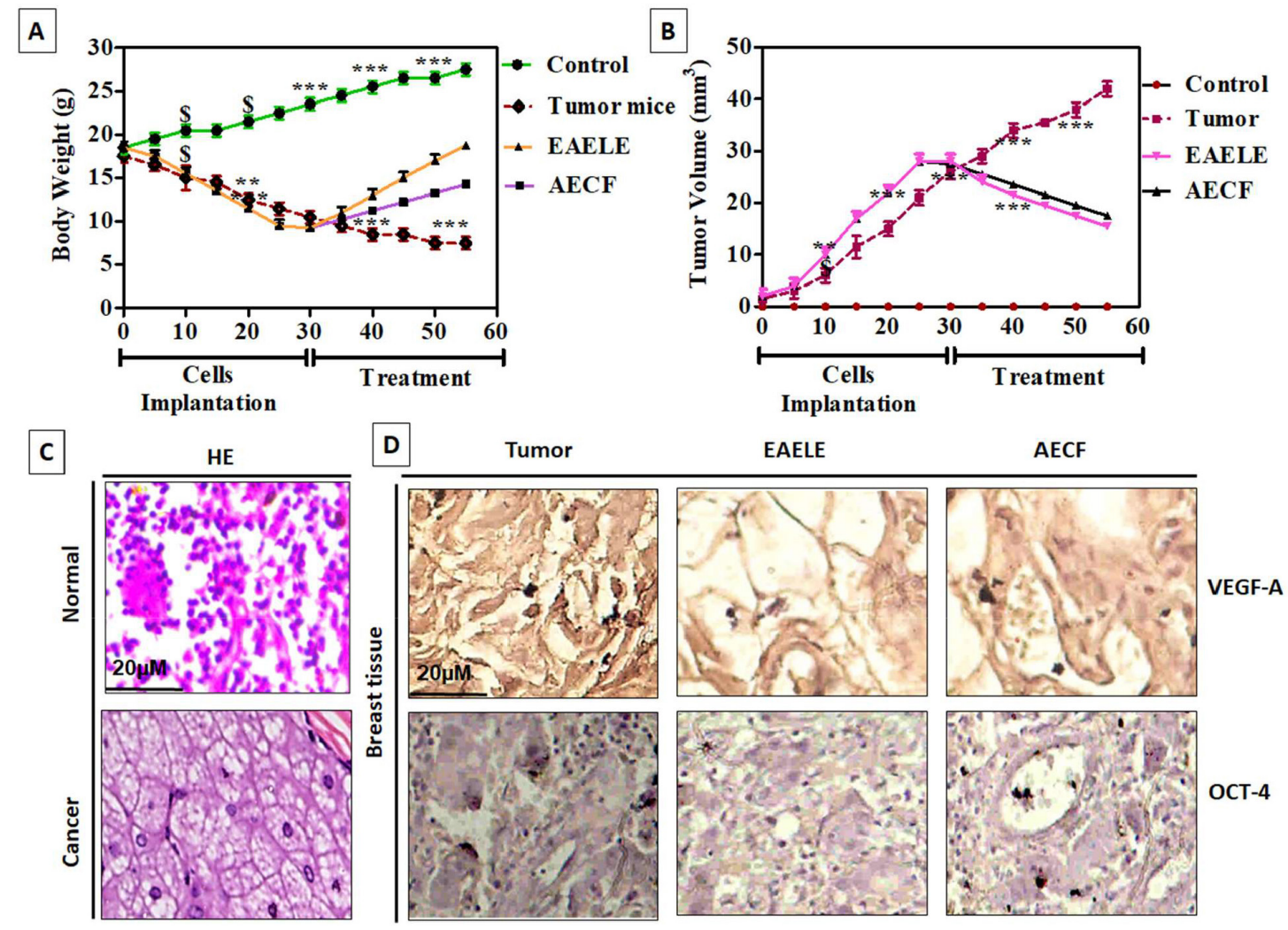

Figure 7: Lycopersicon esculentum fruits and Cassia fistula pods decreased the metastasis and angiogenesis markers in the xenograft mice model. (A) Average body weight of mice before and after treatment with the EAELE and AECF. (B) Average tumour volume of mice. Data were presented as the mean \pm SD. (C) H\&E staining of the xenograft tissue section. Scale bar 20 $\mu \mathrm{m}$. (D) Immunohistochemical expressions of VEGF-A and OCT-4 in different tumour tissues. Image used as the representative of three independent experiments.

Table 1: IC ${ }_{50}$ values of various extracts of Lycopersicon esculentum fruits and Cassia fistula pods on human breast cancer cell line MCF-7. All values are in $\mu \mathrm{g} / \mathrm{ml}$. "-" represents that the $\mathrm{IC}_{50}$ value is above $1000 \mu \mathrm{g} / \mathrm{ml}$.

\begin{tabular}{cccccccccc} 
Cell line & Hours & \multicolumn{3}{c}{$\begin{array}{c}\text { Extracts of Lycopersicon } \\
\text { esculentum fruits }\end{array}$} & \multicolumn{3}{c}{ Extracts of Cassia fistula pods } \\
& & AELE & MELE & BELE & EAELE & AECF & MECF & BECF & EAECF \\
MCF- 7 & 24 & - & - & - & 750 & 350 & 550 & - \\
& 48 & 1000 & 890 & - & 550 & 300 & 450 & - & 830 \\
& 72 & 570 & 560 & 890 & 220 & 150 & 300 & 650 & 660 \\
\hline
\end{tabular}

